\title{
Diffusion performance of moisture in conductive adhesive interconnection structure
}

\author{
Yang Lu \\ Electromechanical Engineering College \\ Guilin University of Electronic Technology \\ Guangxi, China \\ 1067467611@qq.com
}

\author{
Dejian Zhou \\ Electromechanical Engineering College \\ Guilin University of Electronic Technology \\ Guangxi, China \\ emezdj@guet.edu.cn
}

\begin{abstract}
This paper studied on the environment temperature and humidity setting of conductive adhesive in microelectronic packaging, and established DGEBA /MPD linear homo-polymer model which simplified the conductive adhesive based on material Studio software, to access to diffusion mean square displacement curve in the different temperature and humidity water in DGEBA /MPD polymer. Based on the Einstein formula, through the mean square displacement curve to calculate the slope (a) showed that under the same low humidity conditions, the temperature is high, the water diffusion coefficient is larger, the diffusion is faster. According to different humidity conditions, under the same temperature and low humidity conditions, the diffusion coefficient of water molecules increases with the increase of humidity. Correlation algorithm simulated the diffusion of water molecules and it helped to improve the efficiency of the use of conductive adhesive. Relevant analysis is helpful to guide the conductive adhesive interconnect process parameter settings.
\end{abstract}

Keywords-conductive adhesive; DGEBA; MPD; diffusion coefficient; molecular dynamics

\section{INTRODUCTION}

Conductive adhesive interconnection technology is mainly used in the microelectronics package in the bare chip or chip placement and flip chip (Flip-Chip) package. In the component of structure function integration , conductive adhesive bonding method is applied to make up for the interlayer welding, pin and so on . The structure of the chip and the substrate is interconnected by conductive adhesive .conductive adhesive interconnection performance is impacted in external environment. Temperature, humidity, etc can produce effects on the Internet characteristics of conductive adhesive ${ }^{[1]}$. Conductive adhesive has varying degrees of moisture absorption, and the adsorption of water will cause the degradation of the colloid. Too much water can lead to the free volume of the matrix, and the interface will be subjected to the radial tensile stress along the fiber. The swelling of the resin matrix to the large molecule, leading to the increase of the distance between the molecules, which leads to the increase of the activity of the rigid group, so as to increase the plasticity of the matrix, so that the rigidity of the material is reduced. In addition, during the diffusion of water molecules, the micro cracks and micro pores in the resin matrix changed, and the moisture absorption of the matrix increased leads to the breaking of the chain segment. Moisture absorption leads to various problems such as thermal performance, electrical properties and mechanical properties, so that the moisture absorption property of polymer has become a key issue in the field of reliability in microelectronic packaging ${ }^{[2]}$. $\mathrm{Li}$ Hua and other researchers showed that in the condition of the high temperature ( 80 degrees $\left.{ }^{\circ} \mathrm{C}\right)$ and high humidity (RH85\%) aging, electrical characteristics conductive adhesive significantly enhanced ${ }^{[3]}$; Jong et Studied a variety of ACF aging experiments, and also showed that the thermal conductivity and contact resistance of the materials changed significantly in the humid and high temperature environment [4-6]. Therefore, it is necessary to consider the influence of temperature and humidity on the interconnect performance of conductive adhesive interconnect structure. In this study, the diffusion of water molecules was studied, in order to reduce the diffusion capacity of water in the colloid ${ }^{[7]}$. Research the moisture absorption and diffusion of water molecules in the gel can guide the design of temperature and humidity parameters of the interconnect process environment and to ensure the quality of product interconnection.

\section{LINEAR HOMO SYSTEM MODEL OF MPD AND DGEBA}

In this paper, the structure of the chip and the substrate conductive adhesive is studied. External hot and humid environment causes the interconnection structure to appear aging, which affects the Mechanical, electrical and thermal performance. In order to reduce the influence of external factors such as temperature and humidity on the interconnection performance, it is necessary to reduce the diffusion capacity of moisture in the interconnected colloid. In the study of the diffusion properties of moisture in the colloid, a simplified model is drawn out, and the boundary conditions are studied. The diffusion of water in colloid with different concentration was studied, and the diffusion of water molecules under different temperature and humidity conditions are obtained, so as to guide the setting of the process parameters. Use the structure of DGEBA and MPD to characterize the model of conductive adhesive. Molecular dynamics simulation software Studio Material is used, and the modules used include Amorphous, Cell Discover, Visualizer and Forcite and so on.

In this paper, the conductive adhesive model is a threedimensional periodic structure of DGEBA and MPD According to the properties of the three-dimensional periodic 
boundary, the structure of the smallest unit in the system can be studied when the system or model is too large. The structure of the system is shown in Figure 1.Different colors represent different elements. Red represents oxygen, blue represents nitrogen, gray represents carbon, and white represents hydrogen. The lattice parameter of the three dimensional periodic box is $20 * 20 * 20$, and the initial density is $0.98 \mathrm{~g} / \mathrm{cm} 3$. The model contains 6 long chains of Epoxy resin molecular and curing agent manual connection. A long chain contains a DGEBA and two MPD. In this paper, the different temperature and humidity process parameters were 2 , 4, 6 water molecules and 300K, 350K, 400K, $450 \mathrm{~K}$.

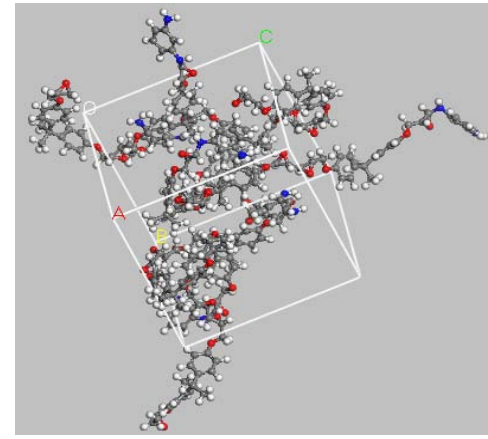

Fig.1. DGEBA and MPD homo - mesh - dimensional periodic model

Study on diffusion properties of water molecules based on DGEBA/MPD model

\section{A. Effect of temperature on the diffusion properties of water molecules}

Three models containing one, two and three water molecules are researched in the atmosphere of $300 \mathrm{~K}, 350 \mathrm{~K}$, $400 \mathrm{~K}, 450 \mathrm{~K}$, diffusion coefficient of water molecules is used to represent diffusion properties of water molecules in polymer. Diffusion of water molecules in polymer is often analyzed after NVT molecular dynamics simulation. In this paper, the Compass force field is used as the potential function, and the diffusion coefficient is calculated by the Einstein formula. The formula is shown as formula 1 :

$$
D=\frac{1}{6 N} \frac{d}{d_{t}} \lim _{t \rightarrow \infty} \sum_{k=1}^{N}\left\langle\left|r_{k}(t)-r_{k}(0)\right|^{2}\right\rangle
$$

$\mathrm{D}$ represents the diffusion coefficient; $\mathrm{N}$ represents the total number of particles; $\left\langle\left|r_{k}(t)-r_{k}(0)\right|^{2}\right\rangle$ represents the mean square displacement.

In Studio Material software, mean square displacement is expressed to study the average value of all the atoms in the three-dimensional box at all time intervals, so the formula 1 can be simplified as $D=\frac{a}{6}$; a indicates the slope of time to mean square displacement.

In this paper, the dynamic simulation of 100ps under the NVT system is carried out, and the mean square displacement curves of these models in $300 \mathrm{~K}, 350 \mathrm{~K}, 400 \mathrm{~K}, 450 \mathrm{~K}$, are obtained. Analysis of the 100ps time step is used $100 \mathrm{fs}$ as a frame output, getting 1000 points to diagram mean square curve with time which are shown in figure 2,3,4.

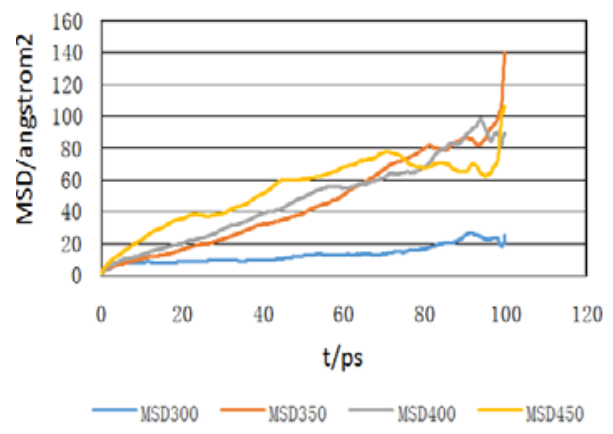

Fig.2. two water molecules model

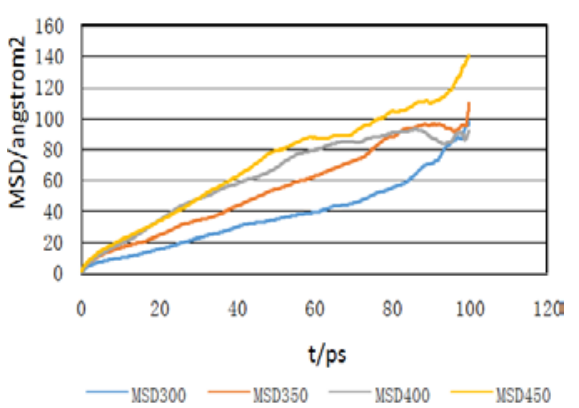

Fig.3. four water molecules model

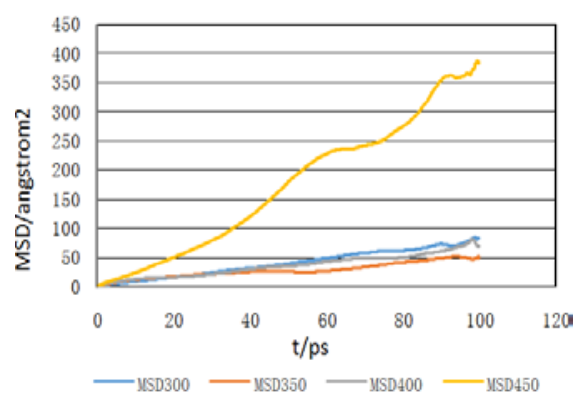

Fig.4. six water molecules model

Curing temperature of conductive adhesive is generally lower than 423K, the temperature range of 300-450K selected in this paper is suitable to study the diffusion mechanism of water. Due to the time of the dynamics simulation of the process simulation is so short that the model structure does not achieve complete balance. Trailing phenomenon happens at the end of the curve reflects the defect. So in order to obtain the feasible mean square displacement of the slope of the time to mean square displacement, select the middle time period to calculate. its specific values are shown in table I.

Table I the values of a under different temperature and humidity

\begin{tabular}{|c|c|c|c|}
\hline $\mathrm{T}$ & 2 water & 4 water & 6 water \\
\hline $30(1 \mathrm{~K}$ & 0.104 & 0.649 & 0.419 \\
\hline $350 \mathrm{~K}$ & 0.690 & 0.938 & 0.633 \\
\hline $400 \mathrm{~K}$ & 0.829 & 1.403 & 0.788 \\
\hline
\end{tabular}


The data in Table 1 show that values of slope a in the model with 2,4,6 water molecules in the simulated four temperature correspond to $0.104,0.690,0.829,1.027$ and $0.649,0.938,1.403,1.408$ and $0.419,0.633,0.788,3.928$. The change of a under the same humidity condition was observed, and it was found that with the increase of temperature, a gradually became larger. According to formula 1, the change law of a along with temperature can be expressed by linear diffusion coefficient along with the change of temperature. The diffusion coefficient of water molecules increases along with the increase of temperature at the same humidity. So in low water absorption of epoxy resin model, in order to ensure the performance of epoxy resin and water molecule diffusion influence on the properties of epoxy resin to minimize, from the point of view of temperature, it needs to choose the lower working temperature of the epoxy resin, meet in the realization of the curing meanwhile makes water molecular diffusion performance drop to the lowest, which can reduce the influence of humidity on the properties of epoxy resin.

\section{B. Effect of humidity on the diffusion properties of water molecules}

In the study of the influence of humidity on the diffusion of water molecules, the simulation results were analyzed in combination with the simulation results of Table 1 . Under the same temperature standard, the change of a value under different humidity (different water molecular quantity) was analyzed. In the case of $300 \mathrm{~K}$, for example, the corresponding values of a are $0.104,0.649,0.419$. It is observed that there are no specific changes in the results. According to the record of the relevant research literature, diffusion properties of water molecules in the structure of epoxy resin need to guarantee that the concentration of water molecules is in the low range of concentration. If it is over this range, water molecules no longer comply with the diffusion regularity of water. $450 \mathrm{~K}$ has exceeded the curing temperature of the polymer, and its curing mechanism also affects the diffusion of water, resulting in the deviation of the slope of Figure 3. Therefore, we select the low concentration (3 water molecules) humidity environment to do a set of simulation experiments. The new control group is shown in Table II.

Table II the value of a under different temperature and humidity

\begin{tabular}{|c|c|c|c|}
\hline 30ิ0 & 2 water & 3 water & 4 water \\
\hline $350 \mathrm{~K}$ & 0.104 & 0.268 & 0.649 \\
\hline $400 \mathrm{~K}$ & 0.829 & 0.774 & 0.938 \\
\hline $450 \mathrm{~K}$ & 1.027 & 1.034 & 1.403 \\
\hline
\end{tabular}

Table II shows that in the case of low moisture content, the diffusion of water molecules is accelerated with the increase of humidity under the same temperature. Therefore, it is important to avoid a large humidity environment in order to avoid increasing the diffusion of water in the epoxy resin and thus reduce its use performance.

\section{Effect of temperature and humidity on water diffusion and optimization}

On the basis of the study of diffusion performance of water influenced by temperature and humidity, we obtained the curve of slope a, as shown in Figure 5, Figure 6.

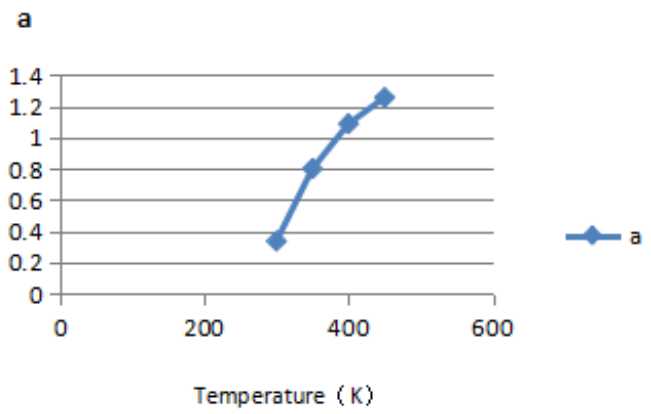

Fig.5. Relationship between temperature and a

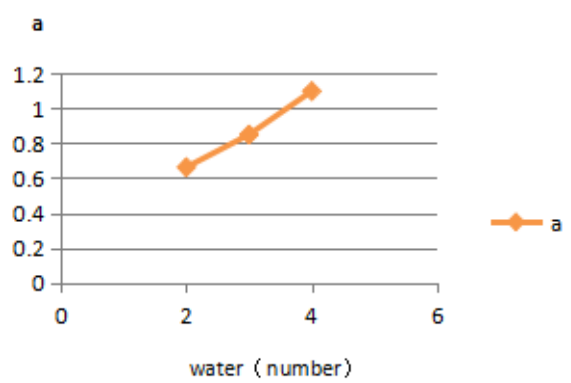

Fig.6. Relationship between humidity and a

The change trend of a from the above diagram shows that with the increase of temperature and humidity, the value of a increases, which indicates that the diffusion coefficient of water becomes larger, and the diffusion of water increases, which is consistent with the results of 2.2 and 2.3. But different process parameters influence the diffusion performance of water, and the effect of temperature on the diffusion performance of water is more prominent. Temperature range in Figure 5 contains the polymer curing temperature. The slope of a in $400 \mathrm{~K}$ or so has a big change, which may be related to the curing of the polymer. So in low humidity and humidity conditions, we select temperature range $390-410 \mathrm{~K}$ to optimize ( $1 \mathrm{~K}$ as the temperature interval), in order to ensure the complete curing of the epoxy resin polymer achieves the minimum temperature of interconnection structure for $398 \mathrm{~K}$. So in the condition of low humidity environment temperature, the temperature should be controlled nearby the temperature in which the slope of a Obviously changes.

\section{CONCLUSION}

Based on the results of the above simulations, the following conclusions are obtained:

1) In the range of a low humidity environment, the diffusion of water molecules in the model of the epoxy resin and curing agent accelerates with the increase of 
the temperature, and accelerates with the increase of the temperature.

2) After saturated moisture absorption, the diffusion performance of water molecules will be weakened, but with the increase of temperature, the overall diffusion performance is still showing a trend of improvement.

3) The effect of temperature on the diffusion properties of water in the epoxy resin is more significant than that of the humidity.

\section{REFERENCES}

[1] Qianqiang He. The meso analysis of moisture and heat expansion and shrinkage of fiber composites[D].Nanjing University of Aeronautics \& Astronautics. 2014.
[2] Dongrong Xin. Study on mechanical properties and interfacial failure mechanism of epoxy resin in hot and humid environment[D].South China University of Technology.2013.

[3] Gorgeous, Xing peng Guo, Jiakuan Yang,Fengming Liu. Electrical conductivity and corrosion behavior of microelectronic interconnects ACF under humid and hot environment[J],journal of chongqing university,2009,33(9):119-125.

[4] KIM J W,LEE Y C,JUNG S B. Reliability of conductive adhesives as a $\mathrm{Pb}$-free alternative in flip-chip application[J].Journal of Electronic Material,2008,37(1):9-11.

[5] Sabzevari SM.Cure kinetics and process modeling of a carbon-fiber thermoplastic-toughened epoxy resin prepreg[D].Wichita State University, 2010.

[6] Jiejing Wang. Study on mechanical properties of anisotropic conductive adhesive film (ACF) [D]. Tianjin University,2008.

[7] Hui Li, van Xiaoping, Shuli Yue, the red, Zhang Zuoquan.Molecular dynamics simulation of the diffusion of water in epoxy resin[J], 2014,31(6):696-700.

[8] Chaofu Wu. Molecular simulation study of cross linked epoxy[D].Hunan University.2007. 